\title{
Studying the Effect of $\beta$-Cyclodextrin in Aqueous Polymerization of Some Acrylate Monomers Bearing Hydrophobic Moiety
}

\author{
Y. Assem* and A.A. Yehia \\ Polymers and Pigments Department, National Research \\ Centre, Dokki, Giza, Egypt.
}

\begin{abstract}
7 HE AQUEOUS radical polymerization of hexyl acrylate (HA), hexyl methacrylate (HMA), dodecyl methacrylate (DMA) and stearyl methacrylate (SMA) using 2,2'-Azobis(2-methylpropionamidine) dihydrochloride (V50) as initiator is reported. Only two monomers were polymerized, HA and HMA with conversion of 10 and $13 \%$, respectively. For DMA and SMA no polymers were found. To increase the polymerization ability of these monomers, $\beta$ Cycodextrin (CD) was used to complex the monomers forming the water soluble host-guest monomer complex. The CD-monomer complex was performed in two different solvents namely, chloroform and water followed by polymerization. ${ }^{1} \mathrm{H}$ NMR proved the formation of the CD-monomer host-guest complex. The polymerization yield was found to be $45,41,33$ and $10 \%$ for HA, HMA, DMA, and SMA, respectively for complexes prepared in chloroform, while for complexes prepared in water, the conversions of polymerization found to be 58,56,50, and $45 \%$ for HA, HMA, DMA, and SMA, respectively.The same results were obtained, when the above complexed monomers were copolymerized with diallydimethyl ammonium chloride (DADMAC) even using conventional radical polymerization or controlled radical polymerization.
\end{abstract}

Keywords: Cyclodextrin, Acrylate monomers, Diallyldimethylammonium chloride, Radical polymerization, Reversible additionfragmentation chain transfer polymerization.

Aqueous polymerizations of hydrophobic monomers could be achieved if the monomers are solubilized via host-guest complexation with water-soluble cyclodextrins (CDs) ${ }^{(1-4)}$. Cyclodextrins are cyclic oligoamyloses. They are able to enclose hydrophobic molecules to form host-guest complexes, where the hydrophobic guest molecule is encapsulated by the cyclodextrin.

Generally $\mathrm{CD}$ has a cyclic structure with special conformation. The hydrophilic hydroxyl groups are directed outside to form a hydrophilic exterior and leaves a hydrophobic cavity of $6.5 \AA^{(5)}$ in diameter. This special structure

\footnotetext{
*Email: ya.assem@nrc.sci.eg
} 
makes CD a good candidate to enclose smaller molecules to form water-soluble host-guest complexes. It was reported that CD could be used as phase transfer catalyst to overcome the solubility barrier in emulsion polymerization of highly water-insoluble monomers ${ }^{(6-11)}$. The proposed mechanism is that water-soluble complex formation among $\mathrm{CD}$ and hydrophobe reduces the kinetic barrier and makes it easier for the monomer to leave the monomer droplet. After arriving at the surface of the growing particle, the monomer is released from the complex and is able to enter the particle. Now the free CD can start its transportation again $^{(8)}$. CD has a lot of potential applications in different fields; modified CD derivatives were used to synthesize a new family of star-shaped poly(ethylene oxide) polymers with CD core, having 14 or 21 arms. The polymerization was found to be living and DOSY experiments confirmed the well-defined structures for the synthesized star-polymers ${ }^{(9)}$.

Ritter and co-workers have demonstrated for several water - insoluble monomers that free radical homopolymerization and copolymerization of CDmonomer complexes in aqueous solution is possible. The free radical homopolymerization of tert-butyl methacrylate - cyclodextrin host-guest complexes ${ }^{(12)}$, the free radical homopolymerization of cyclohexyl and phenyl methacrylate-cyclodextrin host-guest complexes ${ }^{(13)}$, the free radical homopolymerization of $\mathrm{N}$-methacryloyl-1-aminononane-cyclodextrin host guest complexes ${ }^{(14)}$ and the free radical copolymerization of hydrophobic acrylate-cyclodextrin host-guest complexes ${ }^{(15-17)}$ and methacrylate cyclodextrin host - guest complexes ${ }^{(18)}$ were reported.

Synthesis of star polymers with a $\beta$-cyclodextrin (CD) core was also reported by Pereira et $a l^{(19)}$. they used the arm-first, then the core-first strategy. Cationic ring opening polymerisation (CROP) of 2-methyl-2-oxazoline (MeOx) was first initiated by allyl bromide, and then quenched with heptakis(6-deoxy-6amino) $\beta-C D$ in order to get a 7-arm star polymer. Aqueous reversible additionfragmentation transfer (RAFT) polymerization of N,N- dimethylacrylamide (DMAAm), N,N-diethylacrylamide (DEAAm), and N-isopropylacrylamide (NIPAAm) utilizing host/guest complexes of cyclodextrin and hydrophobic chain transfer agents (CTAs) at $25^{\circ} \mathrm{C}$ was reported by Bernhard et al. ${ }^{(4)}$. Three novel guest- functionalized CTAs, namely 4-(tert-butyl) phenyl 2-(((ethylthio) carbonothioyl) thio) -2- methylpropanoate, bis (4-tert-butyl) benzyl carbonotrithioate, and benzyl (3-((4-(tert-butyl) phenyl) amino) -3-oxopropyl) carbonotrithioate, were synthesized and employed in aqueous RAFT polymerizations. The presented technique allows for the facile preparation of hydrophilic polymers with hydrophobic end groups in aqueous environments ${ }^{(20)}$.

The aim of this article is to find out the influences of $\beta$-Cyclodextrin in the polymerization of different hydrophobic acrylate derivatives in aqueous medium. The experiments are carried out with Hexyl acrylate (HA), Hexyl methacrylate (HMA), Dodecyl methacrylate (DMA) and Stearyl methacrylate 
(SMA). Furthermore, copolymerization of these monomers with Diallyldimethylammonium chloride (DADMAC) either by conventional free radical polymerization or reversible addition-fragmentation chain transfer polymerization (RAFT) to get random copolymers or amphiphilic copolymers bearing positive charge that has potential applications in different fields.

\section{Materials}

\section{Experimental}

The monomers

Hexyl acrylate (HA), Hexyl methacrylate (HMA), Dodecyl methacrylate (DMA), and Stearyl methacrylate (SMA), were purchased from Sigma-Aldrich and purified by passing over basic alumina column. Diallyldimethylammonium chloride (DADMAC) 65\% water solution, the water soluble initiator 2,2'Azobis(2-methylpropionamidine) dihydrochloride (V 50) were purchased from Sigma-Aldrich and used as received. Beta Cyclodextrin was obtained from Wacker-Chemie Germany. All used solvents are distilled before used, deionized water was used in all experiments. RAFT agent: 3-Benzyltrithiocarbonyl propionic acid was prepared according to the literature ${ }^{(21)}$ HNMR chart is presented in Fig. 5.

\section{Complexation of the monomers with $\beta$-CD}

The used ratio of monomer to $\beta-C D$ was $1: 1$. The specified amount of $C D$ (50 mmol) was dissolved in $\mathrm{CHCl}_{3}$ or $\mathrm{H}_{2} \mathrm{O}$ and left to stir for complete dissolution, sonication for $10 \mathrm{~min}$ is used when water used as a solvent. Then monomers $(50 \mathrm{mmol})$ were added to the cloudy mixtures and left to stir at room temperature for $24 \mathrm{hr}$. The solid product was filtered and dried until constant weight. Yield was determined gravimetrically (Method A, Scheme 1). A third experiment was done, where monomers were complexed with $\mathrm{CD}$ in water and stirred for $30 \mathrm{~min}$, then the initiator was added and polymerization started at 70 ${ }^{\circ} \mathrm{C}$ for $3 \mathrm{hr}$ (Method B, Scheme 1).

\section{Radical polymerization in aqueous media}

The CD-monomer complex solid was charged in a $50 \mathrm{ml}$ schlenk flask containing magnet bar and $5 \mathrm{ml}$ deionized water. V50 initiator was added so that final monomer: initiator is 100:1. The reaction solution was purged with Ar for $20 \mathrm{~min}$. The flask was then placed in an oil bath at $70{ }^{\circ} \mathrm{C}$ for $4 \mathrm{hr}$. After that time, the obtained polymer was purified by precipitation in methanol to get a white solid that is dried in a vacuum oven at $40{ }^{\circ} \mathrm{C}$.

For RAFT polymerization, the aforementioned procedures were repeated and RAFT agent was added in a threefold amount to initiator concentration. The reaction was continued for $10 \mathrm{hr}$. At the end of the reaction, DADMAC and V50 were charged to the flask and the reaction proceeded further at $70{ }^{\circ} \mathrm{C}$ for $10 \mathrm{hr}$. Purification of the polymer was done by precipitation in methanol/acetone mixture in $1: 2$ 


\section{Characterization}

Gel permeation chromatography (GPC)

The molecular weights of all polymers were measured by Chloroform GPC at $25^{\circ} \mathrm{C}$. Sample concentration was $1 \mathrm{~g} \mathrm{~L}^{-1}$. The apparatus consists of a Gynkotek HPLC pump, an Agilent Auto-sampler 1200, linear columns (PSS, polystyrene) consisting of a pre-column $10 \mu, 8$ x $50 \mathrm{~mm}$, a column $10 \AA, 8 \mathrm{x}$ $300 \mathrm{~mm}$ and two columns $3000 \AA$, 8 x $300 \mathrm{~mm}$. As detector, RI detector of Knauer Company was used. As calibration, linear PMMA with a narrow mass distribution and molecular weight between 500 and 1,000,000 was used. Toluene was used as an internal standard. The elugram was evaluated with PSS WinGPC Unity program.

Nuclear magnetic resonance spectroscopy (NMR)

NMR spectra were recorded on a Bruker DRX-300 spectrometer using Tetramethylsilane (TMS) as an internal standard and $\mathrm{CDCl}_{3}$ as a solvent.

\section{Results and Discussion}

Radical polymerization of the four monomers in aqueous media

The reactions were carried out in water at $70{ }^{\circ} \mathrm{C}$ for $4 \mathrm{hr}$. Only HA and HMA were polymerized with low yield, while the DMA and SMA (Fig. 1) could not be polymerized directly in water due to the heterogeneity of the polymerization system as shown in Table 1 . The less solubility of SMA, DMA and HA in water is turned out by the less amount of received polymer. HA is less soluble in aqueous phases than HMA ${ }^{(15)}$. SMA and DMA are completely insoluble in water. So there is no polymer formed of SMA and DMA.

TABLE 1. Radical polymerization of the hydrophobic monomers in $\mathrm{H}_{2} \mathrm{O}$.

\begin{tabular}{cc}
\hline Monomer & Polymer Conversion [\%] \\
\hline PHA & 10 \\
PHMA & 13 \\
PDMA & --- \\
PSMA & -- \\
\hline
\end{tabular}


<smiles>C=CC(=O)OCC</smiles>

\section{Hexyl acrylate} $\mathrm{C}_{9} \mathrm{H}_{16} \mathrm{O}_{2}$ $\mathrm{M}=156.25 \mathrm{~g} / \mathrm{mol}$<smiles>C=C(C)C(=O)OCC</smiles>

Hexyl methacrylate $\mathrm{C}_{10} \mathrm{H}_{18} \mathrm{O}_{2}$ $M=170.25 \mathrm{~g} / \mathrm{mol}$<smiles>C=C(C)C(=O)OCC(C)(C)C</smiles><smiles>C=C(C)C(=O)OCC(C)(C)I</smiles>

Dodecyl methacrylate $\mathrm{C}_{16} \mathrm{H}_{30} \mathrm{O}_{2}$ $\mathrm{M}=254.42 \mathrm{~g} / \mathrm{mo}$
Stearyl methacrylate

$$
\mathrm{C}_{22} \mathrm{H}_{42} \mathrm{O}_{2}
$$

$\mathrm{M}=338.58 \mathrm{~g} / \mathrm{mol}$<smiles>CC(C)(N=NC(C)(C)C(=N)N)C(=N)N</smiles>

2,2'-Azobis(2-methylpropionamidine) dihydrochloride $\mathrm{C}_{8} \mathrm{H}_{20} \mathrm{~N}_{6} \mathrm{Cl}_{2}$ $\mathrm{M}=271.19 \mathrm{~g} / \mathrm{mol}$

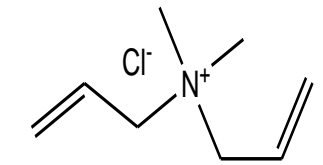

Diallyldimethylammonium chloride

$$
\mathrm{C}_{8} \mathrm{H}_{16} \mathrm{NCl}
$$

$\mathrm{M}=161.67 \mathrm{~g} / \mathrm{mo}$<smiles>O=C(O)CCSC(=S)SCc1ccccc1</smiles>

3-Benzyltrithiocarbonyl propionic acid

$$
\begin{gathered}
\mathrm{C}_{11} \mathrm{H}_{12} \mathrm{O}_{2} \mathrm{~S}_{3} \\
M=272.41 \mathrm{~g} / \mathrm{mol}
\end{gathered}
$$

\section{Fig.1. Structure of monomers, initiator and RAFT agent used in polymerization.}

\section{Complexation of the monomers with $\beta$-CD in $\mathrm{CHCl} 3$ within $24 \mathrm{hr}$}

The used ratio of monomer to $\beta-C D$ was $1: 1$. The monomers were added to $\mathrm{CHCl}_{3}$. The cloudy reaction mixtures were stirred at RT for $24 \mathrm{hr}$. The precipitated monomers were filtered off and dried at $40{ }^{\circ} \mathrm{C}$ in vacuum to give an 
excellent yield which is mostly close to $100 \%$. The obtained CD-monomer complexes were polymerized in water at $70{ }^{\circ} \mathrm{C}$ for $4 \mathrm{hr}$. Scheme 1 represents the steps of the complexation and polymerization. The conversion of obtained polymers ranged from $10 \%$ in case of PSMA to $45 \%$ for PHA. It is noted that the conversion is decreased as the length of the hydrophobic chain increased. The same behaviour also is observed with the molecular weight of the polymers as shown in Table 2. It is expected that as the length of the hydrophobic chain increases, it is coiled and the propagating radical finds difficulty to diffuse through the polymer chains, consequently the molecular weight will decrease. Polydispersity index is in the normal range of the conventional radical polymerization. GPC elugrams are illustrated in Fig. 2 and they are all monomdal, no shoulders or any monomeric residue. NMR of DMA either free or complexed and PDMA as a representative example is shown in Fig. 3. In this figure a moiety of the $\mathrm{CD}$ protons are appeared clearly in the region of 3-4 ppm, while the vinyl protons at 5.3 and $6 \mathrm{ppm}$ are totally disappeared in the PDMA.
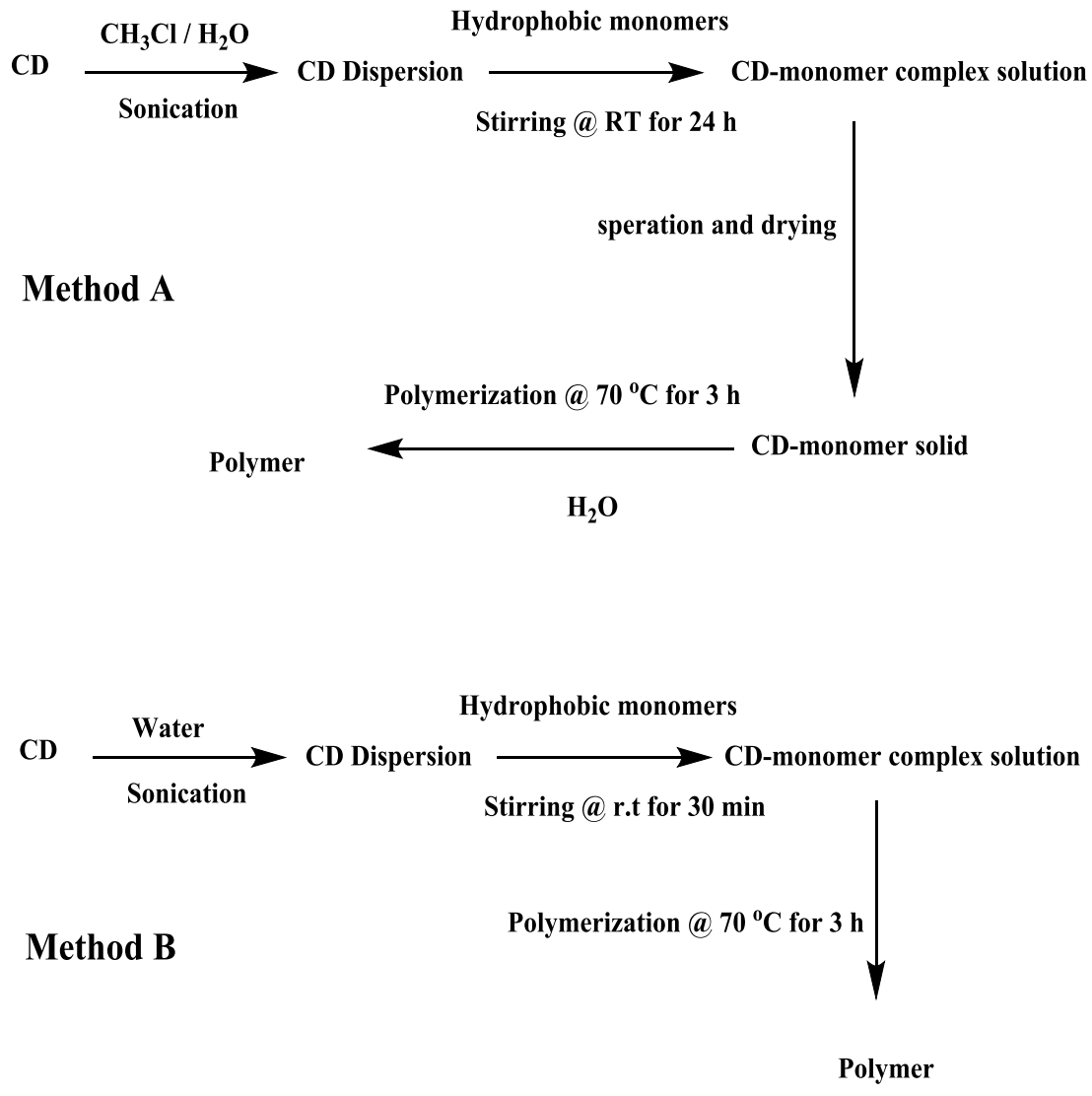

Scheme 1. Methods of CD-monomer complexation . 
TABLE 2. Conversion, molecular weight and PDI of monomer-CD complex in chloroform.

\begin{tabular}{ccccc}
\hline Monomer-CD Complex & Conversion \% & $\begin{array}{c}\text { Polymer } \\
\text { Conversion \% }\end{array}$ & Mn & PDI \\
\hline HA & 98 & 45 & 14500 & 2.20 \\
HMA & 97 & 41 & 15100 & 2.15 \\
DMA & 98 & 33 & 14100 & 2.17 \\
SMA & 99 & 10 & 13200 & 2.22 \\
\hline
\end{tabular}

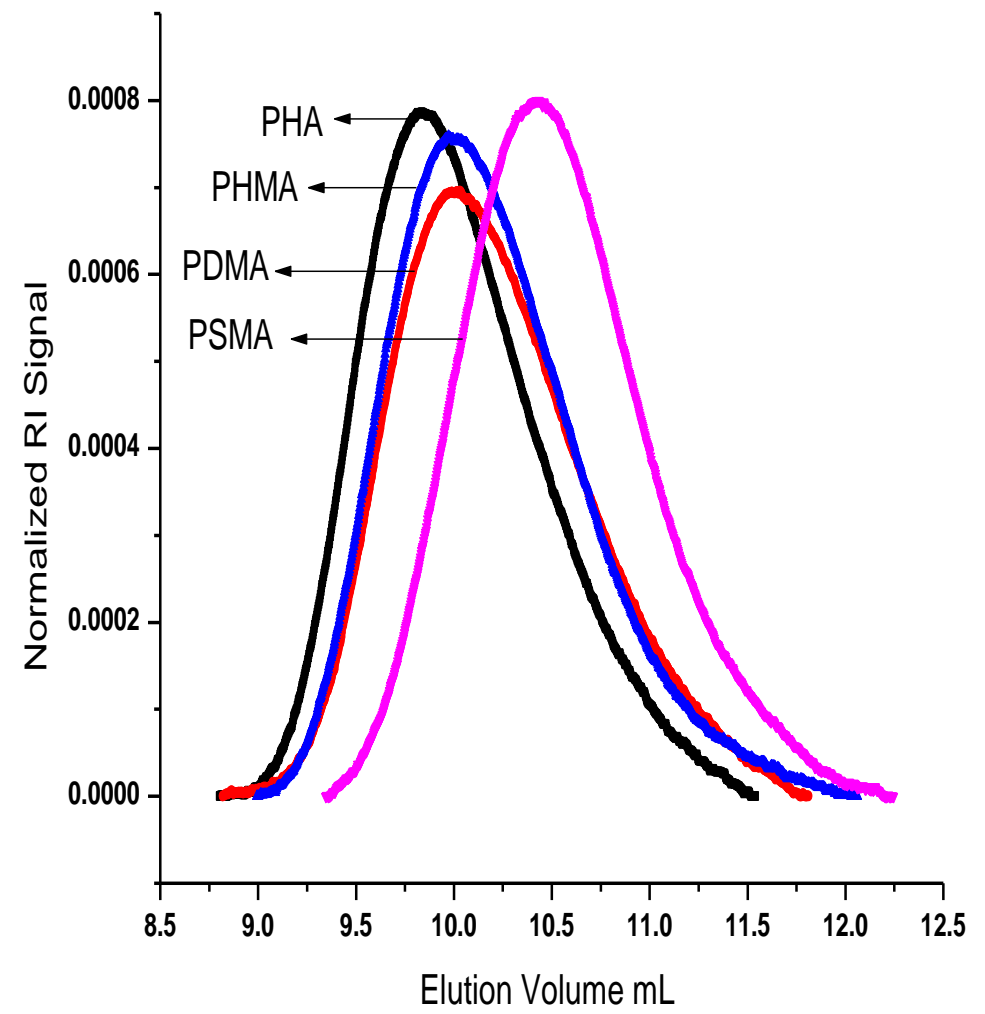

Fig.2. GPC elugram of the four different polymers in Table 1. 


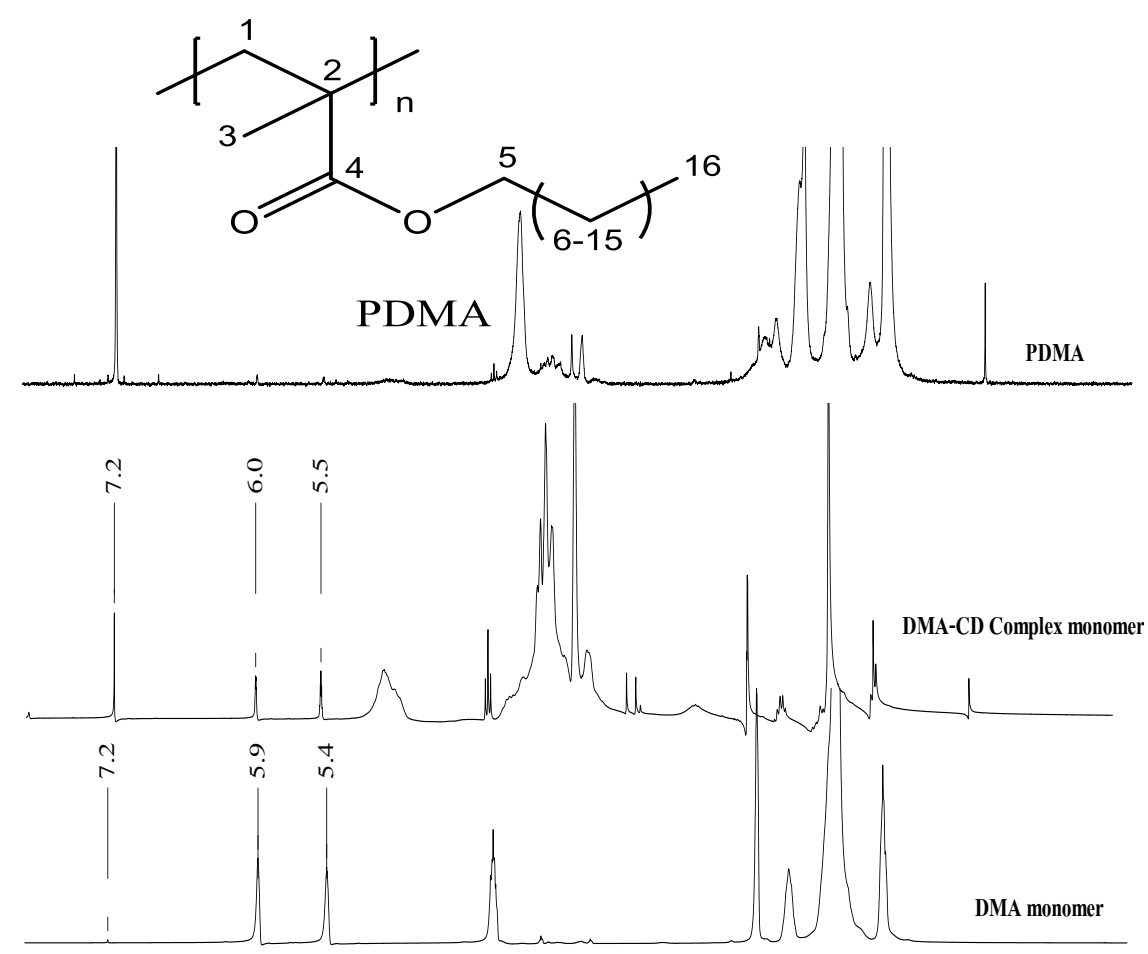

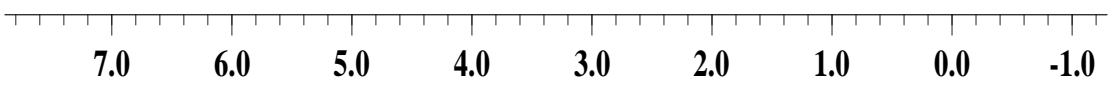

ppm

PDMA: ${ }^{1} \mathrm{H}-\mathrm{NMR}\left(300 \mathrm{MHz}, \mathrm{CDCl}_{3}\right): 3.91(\mathrm{~m}, 2 \mathrm{H}, 5-\mathrm{H}) ; 1.56(\mathrm{~m}, 3 \mathrm{H}, 16-\mathrm{H}) ; 1.27$ (m, 20H, 6-15-H); 0.89 (m, 5H, 1,3-H) ppm.

Fig. 3. An overly of ${ }^{1} \mathrm{H}$ NMR of free DMA, DMA-CD complex and PDMA.

Complexation of the monomers with $\beta-C D$ in water

The used ratio of monomer to $\beta-C D$ was $1: 1$. The monomers were added to water. The cloudy reaction mixtures were stirred at $70{ }^{\circ} \mathrm{C}$ for $24 \mathrm{hr}$. Conversion and the results of the polymerization are summarized in Table 3 . The obtained Monomer-CD complexes are also of high yield. Conversion and molecular weights of the obtained polymers are higher than those complexed in chloroform. The yield represents the amount of the obtained monomer-CD complexes, while the conversion represents how much monomer converted to polymer. The same experiments were done with less complexation time, i.e. after only $30 \mathrm{~min}$. In this case the initiator was added directly at the end of complexation time $(30 \mathrm{~min})$ and the polymerization took place for $4 \mathrm{hr}$. The

Egypt. J. Chem. 58, No. 3 (2015) 
results are represented in Table 4. The results in this case is still accepted, where the conversion and molecular weights of the obtained polymers are higher than those in chloroform taking into consideration that only $30 \mathrm{~min}$ are enough to complex the monomer to CD. Figure 4 shows the conversions of the obtained polymers in different complexation conditions. Water is a more suitable than chloroform to form the monomer-CD complexes, and one hour a time of complexation is enough to assure the formation of monomer-CD complexes with very good yield.

TABLE 3. Conversion, molecular weight and PDI of monomer-CD complex in $\mathrm{H}_{2} \mathrm{O}$ (24 hr).

\begin{tabular}{ccccc}
\hline $\begin{array}{c}\text { Monomer } \\
\text { complexed with } \boldsymbol{\beta}-\end{array}$ & $\begin{array}{c}\text { Yield } \\
\text { CD }\end{array}$ & $\begin{array}{c}\text { Polymer } \\
\text { Conversion } \%\end{array}$ & Mn & PDI \\
\hline HA & 93 & 58 & 17500 & 2.04 \\
HMA & 92 & 65 & 15200 & 2.10 \\
DMA & 96 & 54 & 15100 & 2.10 \\
SMA & 90 & 51 & 12200 & 2.20 \\
\hline
\end{tabular}

Because of the solubility of all monomers in $\mathrm{CHCl}_{3}$, the complexation of monomers is not preferred. To increase complexation rate the monomers were complexed in a solvent in which they are insoluble. The complexation of all monomers in water within $24 \mathrm{hr}$ shows a significant increase of yielded polymers. The yields of PDMA, PSMA, PHA and PHMA are all over 50\% as represented in Table 3. To speed up polymerization the time of complexation is reduced to $30 \mathrm{~min}$. The yields of received polymers are decreasing around $10 \%$ but in a polymerization which takes only $4.5 \mathrm{hr}$ (time of complexation and polymerization time) instead of $28 \mathrm{hr}$. Only the amount of PSMA decreases to $35 \%$.

TABLE 4. Conversion, molecular weight and PDI of monomer-CD complex in $\mathrm{H}_{2} \mathrm{O}$ $(0.5 \mathrm{hr})$.

\begin{tabular}{cccc}
\hline $\begin{array}{c}\text { Monomer complexed with } \\
\boldsymbol{\beta}-\mathbf{C D}\end{array}$ & $\begin{array}{c}\text { Polymer } \\
\text { Conversion } \%\end{array}$ & Mn & PDI \\
\hline HA & 48 & 15400 & 2.21 \\
HMA & 63 & 18400 & 2.30 \\
DMA & 43 & 15500 & 2.21 \\
SMA & 35 & 14500 & 2.32 \\
\hline
\end{tabular}




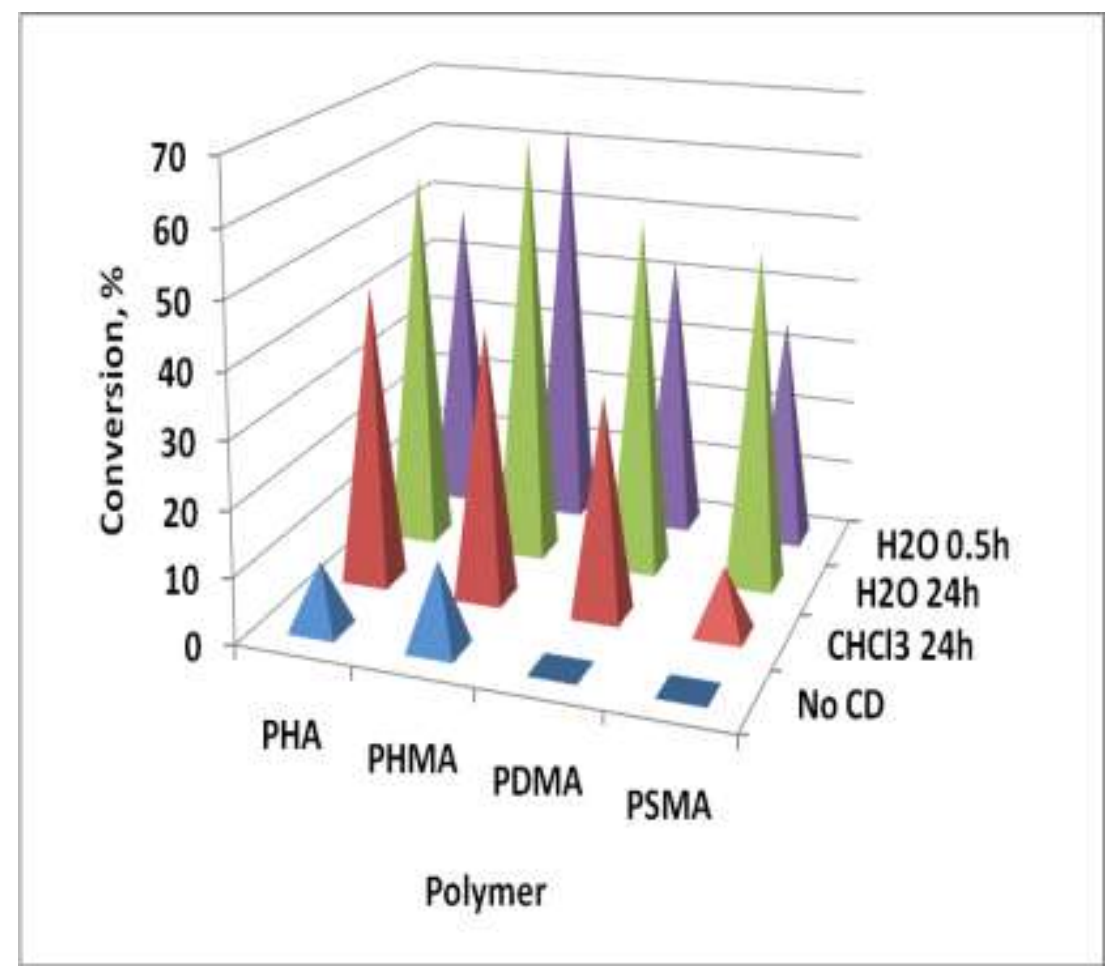

Fig. 4. Conversion of the obtained polymers in different media .

\section{Copolymerization with complexed monomers}

The monomer-CD complexes were copolymerized with DADMAC to get amphiphilic cationic polymers that have a lot of applications. The polymerization was performed under the same polymerization conditions. Two polymerization techniques were applied, the conventional free radical polymerization (FRP) and controlled radical polymerization (CRP) via RAFTmethod. For this purpose an extra mediating agent is synthesized (RAFT agent) as shown in NMR chart in Fig.5. FRP method will produce random copolymers, while RFAT polymerization should produce block copolymers with welldefined architecture as represented in Scheme 2. The obtained results are given in Table 5. The copolymers obtained via FRP are of moderate molecular weight and PDI in the normal range. While those obtained via RAFT mechanism have less values of molecular weights and the PDIs values are out of the range of controlled radical polymerization (normally PDI is less than 1.5). These mean that the obtained polymer via RAFT is not controlled, where the Macro-RAFT monomer-CD complex is not able to initiate the polymerization of DADMAC due to the thermodynamic instability of primary radical of a propagating DADMAC unit according to the mechanism of RAFT polymerization in Scheme 2. 

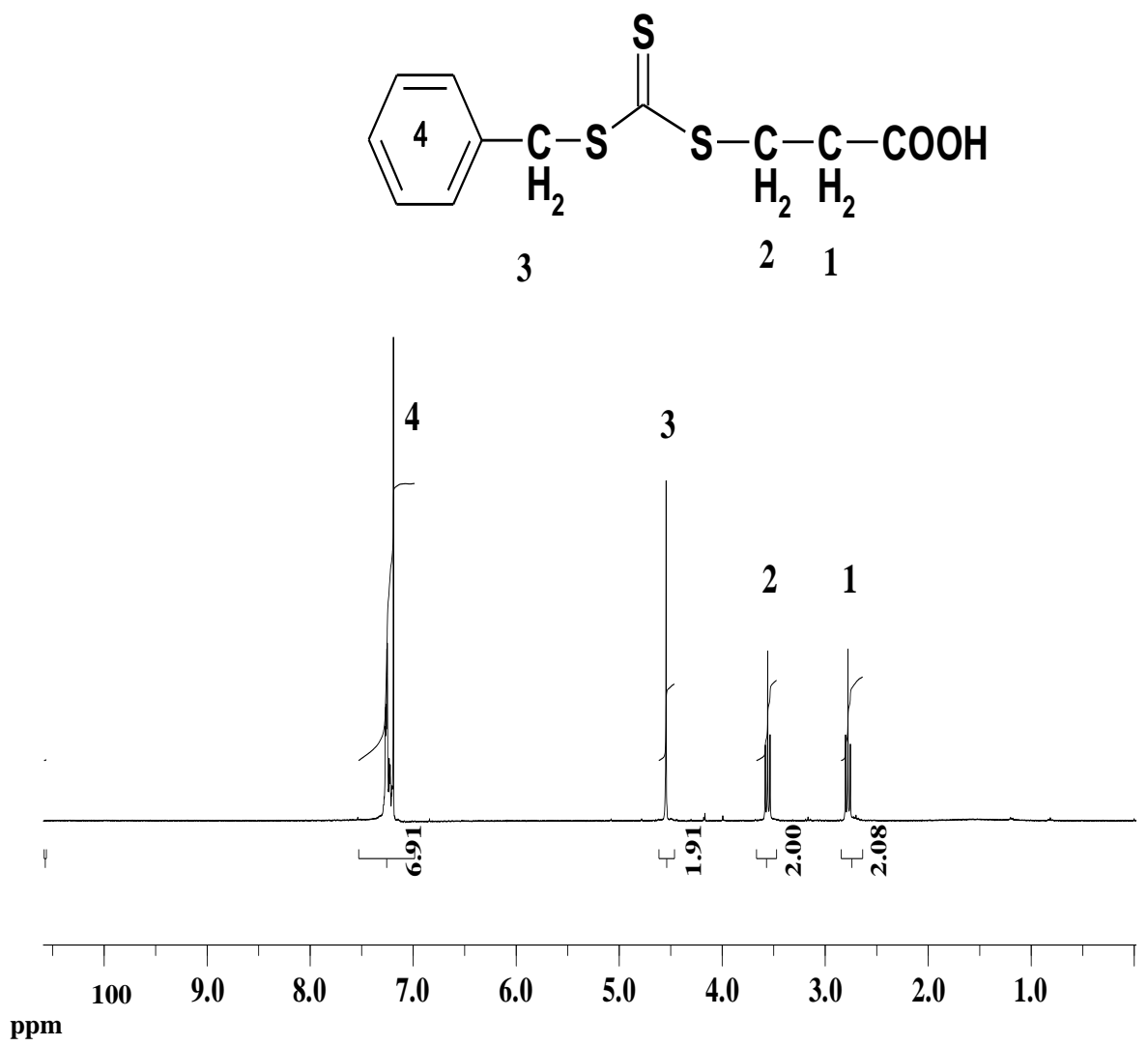

Fig.5. ${ }^{1} \mathrm{H}$ NMR of 3-Benzyltrithiocarbonyl propionic acid used as RAFT agent.

TABLE 5. Conversion, molecular weight and PDI of monomer-CD complex obtained via FRP or RAFT polymerization.

$\begin{array}{lll}\text { Conversion \% } & \text { Mn } & \text { PDI }\end{array}$

Monomer-CD

\begin{tabular}{llllll}
\hline FRP & RAFT & FRP & RAFT & FRP & RAFT
\end{tabular}

\begin{tabular}{ccccccc}
\hline HA & 48 & 40 & 15400 & 14000 & 2.21 & 1.82 \\
HMA & 63 & 50 & 18400 & 11500 & 2.30 & 1.81 \\
DMA & 43 & 30 & 15500 & 12000 & 2.21 & 1.89 \\
SMA & 35 & 18 & 14500 & 11000 & 2.32 & 2.05 \\
\hline
\end{tabular}

Egypt. J. Chem. 58, No. 3 (2015) 

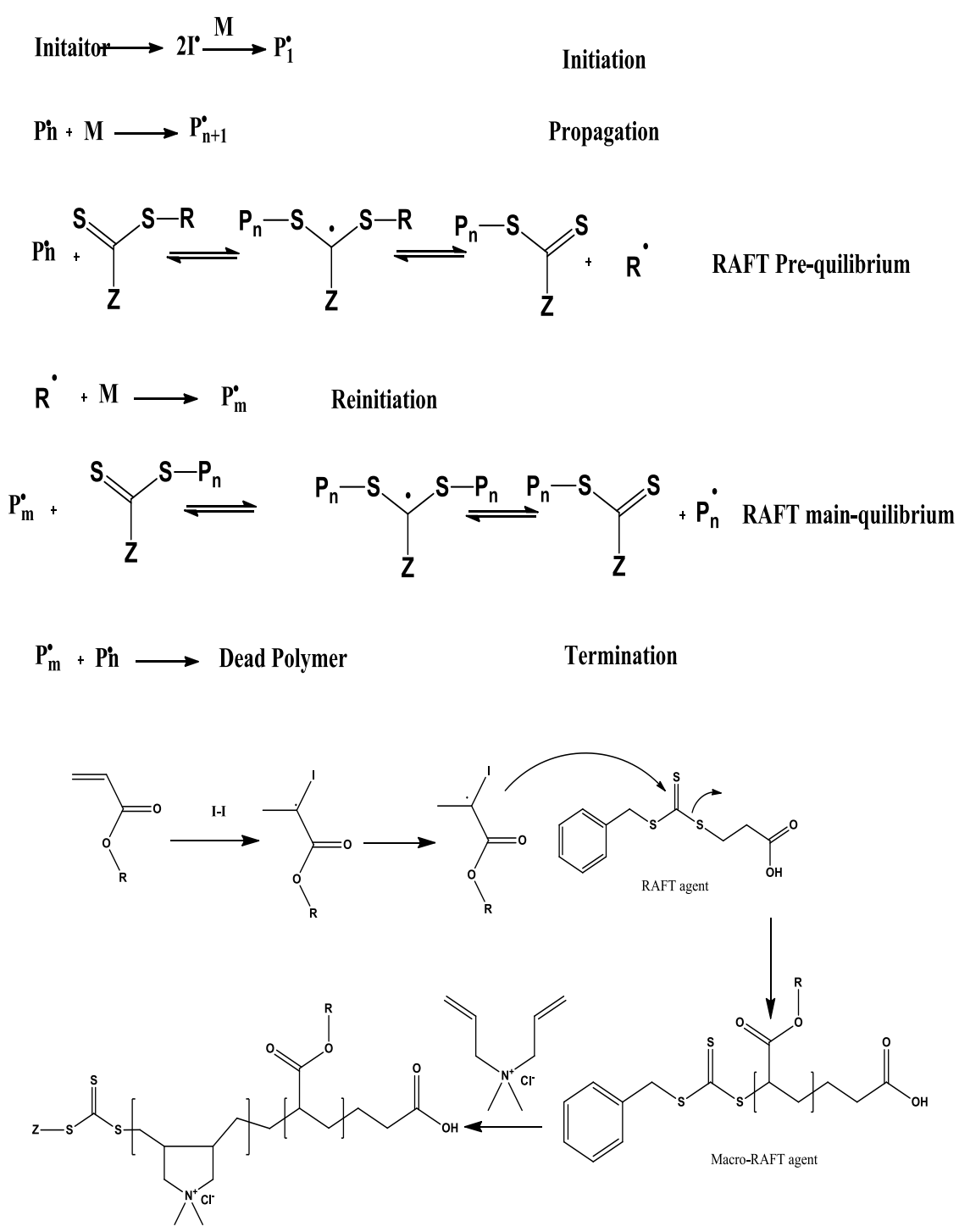

Scheme 2. Proposed RAFT mechanism.

\section{Conclusion}

1. The complex formation of CD with the hydrophobic monomers HA, HMA, DMA and SMA was successfully applied to improve the polymerization of these monomer in aqueous media.

2. The complexation process improved the polymerization of HA and HMA to give higher conversion than when polymerized in water. 
3. For DMA and SMA could not be polymerized in water directly, but with CD complex PDMA and PSMA were obtained with somewhat lower conversion

4. Complexation in water is favoured and $1 \mathrm{hr}$ is enough to form the monomerCD complex.

5. Copolymerization with DADMAC is achieved via free radical polymerization rather than RAFT polymerization due to the thermodynamic instability of RAFT-DADMAC adduct.

\section{References}

1. Wahl, A., Schnell, I. and Pyell, U., Capillary electrochromatography with polymeric continuous beds synthesized via free radical polymerization in aqueous media using derivatized cyclodextrins as solubilizing agents . Journal of Chromatography A, 1044, 211 (2004).

2. Steffens, C. Choi, S. W. and Helmut R., Kinetic studies upon the influence of cyclodextrin on the polymerization of methacrylamides in homogenous aqueous solution. Macromol. Rapid Commun. 27, 542(2006).

3. Schmitz, S. and Ritter, H., Unusual solubility properties of polymethacrylamides as a result of supramolecular interactions with cyclodextrin. Angew. Chem. Int. Ed. 44, $5658-5661$ (2005).

4. Bernhard, V.K., Schmidt, J., Hetzer, M., Helmut, R. and Barner-Kowollik., C., Complex macromolecular architecture design via cyclodextrin host/guest complexes. Prog. Polym . Sci. 39, 235-249 (2014).

5. Rimmer, S., Cyclodextrins in the emulsion polymerization of vinyl monomers . Macromol. Symp. 150,149 (2000).

6. Rimmer, S. and Tattersall, $\mathbf{P}$., The inclusion of $\beta$ cyclodextrin provides supramolecular solution to the problem of polymerization of dodecyl and octadecyl methacrylates in aqueous emulsion. Polymer, 40, 5729 (1999).

7. Rimmer, S. and Tattersall, P.I., Emulsion polymerizations in the presence of $\beta$ cyclodextrin. Polymer, 40, 6673 (1999).

8. Leyrer, R.J. and Machtle, W., Emulsion polymerization of hydrophobic monomers like stearyl acrylate with cyclodextrin as a phase transfer agent. Macromol. Chem. Phys. 201, 1235 (2000) .

9. Huin, C., Nezha, Z. E., Aurica, B., Véronique, F. and Guégan, P., Anionic ring-opening polymerization of ethylene oxide in DMF with cyclodextrin derivatives as new initiators. Carbohydrate Polymers, 94, 323-331 (2013).

10. Lau, W, Emulsion polymerization of hydrophobia monomers. Macromol. Symp, 182, 283 (2002).

11. Sotrsberg, J. and Helmut R., Cyclodextrins in polymer synthesis: A "green" route to fluorinated polymers via cyclodextrin complexes in aqueous solution. Macromol Chem. Phys. 203,812 (2002). 
12. Schwarz-Baraæ S. and Ritter, H., Cyclodextrins in polymer synthesis: Free radical polymerization of a tert-butylmethacrylate-cyclodextrin host-guest system in aqueous medium. J. Macromol. Sci. 40, 437(2003).

13. Jeromin, J., Ritter, H. and Macromol., Cyclodextrins in polymer synthesis: Free radical polymerisation of cyclodextrin complexes of cyclohexyl and phenyl methacrylate in aqueous medium. Macromol. Rapid Commun. 19, 377-379 (1998).

14. Jeromin, J., Noll, O. and Ritter, H., Cyclodextrins in polymer synthesis: Free radical polymerization of cyclodextrin complexes with $N$-methacryloyl-11aminoundecanoic acid or $N$-methacryloyl-1-aminononane as guest monomers. Macromol. Chem. Phys. 199, 2641(1998).

15. Bernhardt, S., Glöckner, P., Theis, A. and Ritter, H., Cyclodextrins in polymer synthesis: Influence of acrylate side groups on the initial rate of radical polymerization of various acrylate/methylated $\beta$-cyclodextrin complexes in water. Macromolecules , 34,1647(2001).

16. Glöckner, P. and Ritter, H., Cyclodextrins in polymer chemistry: Influence of methylated $\beta$-cyclodextrin as host on the free radical copolymerization reactivity ratios of isobornyl acrylate and butyl acrylate as guest monomers in aqueous medium. Macromol. Rapid Commun. 20, 602 (1999).

17. Bernhardt, S., Glöckner, P. and Ritter, H., Cyclodextrins in polymer synthesis: Influence of methylated $\beta$-cyclodextrin as host on the free radical copolymerization reactivity ratios of hydrophobic acrylates as guest monomers in aqueous medium. Polym. Bull. 46,153(2001).

18. Casper, P., Glöckner, P. and Ritter, H., Cyclodextrins in polymer synthesis: Free radical copolymerization of methylated $\beta$-cyclodextrin complexes of hydrophobic monomers with N-Isopropylacrylamide in aqueous medium. Macromolecules, 33, 4361(2000).

19. Guillaume Pereira, Cécile Huin, Simona Morariu, Véronique Bennevault-Celton, and Philippe Guégan, Synthesis of poly(2-methyl-2-oxazoline) star polymers with a $\beta$-cyclodextrin core. Australian Journal of Chemistry, 65 (8), 1145-1155 (2012) .

20. Bernhard V. K. J. Schmidt, Martin Hetzer, Helmut Ritter, and BarnerKowollik, C., Cyclodextrin-complexed RAFT agents for the ambient temperature aqueous living/controlled radical polymerization of acrylamido monomers. Macromolecules, 44, 7220 (2011).

21. Jesberger, M., Barner, L., Stenzel, M. H., Malmström, E., Davis, T. P. and Barner-Kowollik, C. J., Hyperbranched polymers as scaffolds for multifunctional reversible addition-fragmentation chain-transfer agents: A route to polystyrenecore-polyesters and polystyrene-block-poly(butyl acrylate)-core-polyesters. Polym Sci. Part A: Polym. Chem. 41, 3847 (2003). 


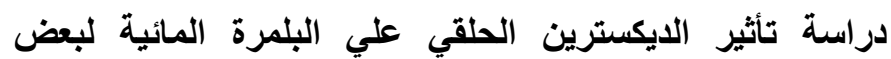 مونومرات الاكريلات المحتوية علي شوارد كاره للماء

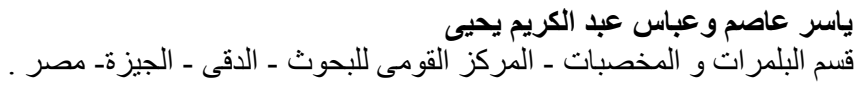

تم بلمرة بعض مونومرات الاكيرالت المحتوية على سلاسل كربونية كاره للماء لمباء

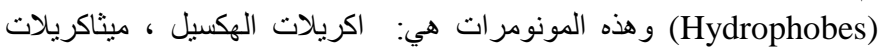

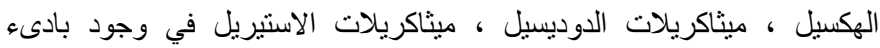

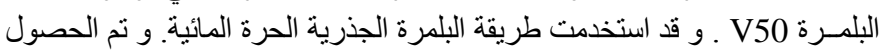

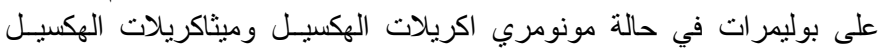

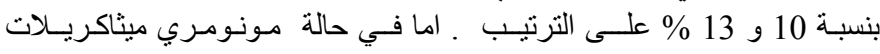

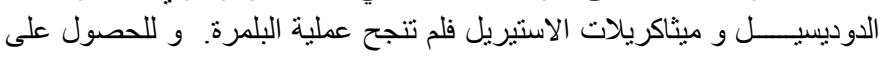

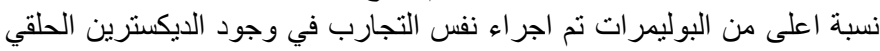

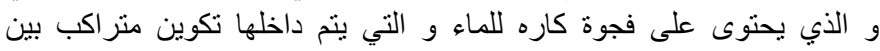

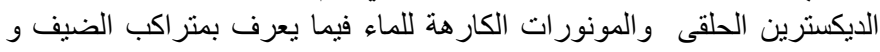

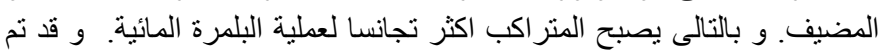

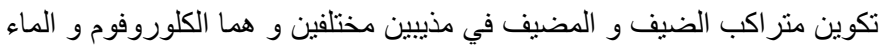

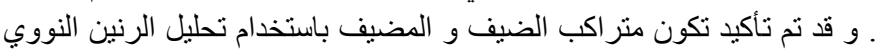

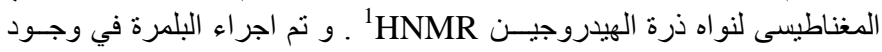

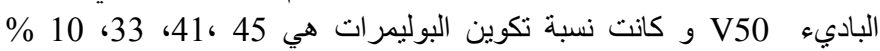

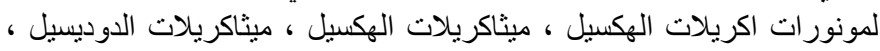

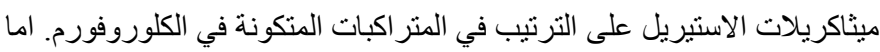

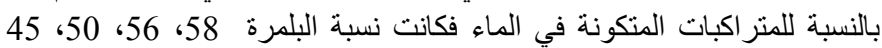

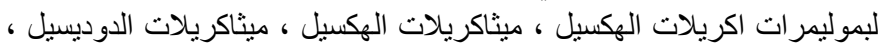

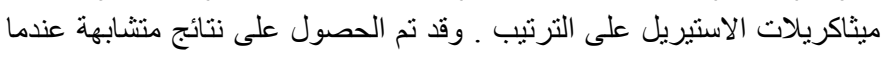

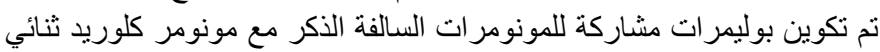

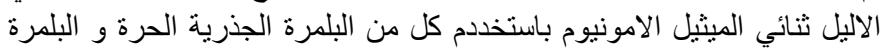

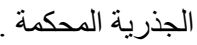


